\title{
Uncovering the social interaction network in swarm intelligence algorithms
}

\author{
Marcos Oliveira ${ }^{1 *}$, Diego Pinheiro ${ }^{2}$, Mariana Macedo $^{3}$, Carmelo Bastos-Filho $^{4}$ and Ronaldo Menezes ${ }^{3}$
}

\author{
*Correspondence: moliveira@tuta.io \\ ${ }^{1}$ Computational Social Science, \\ GESIS-Leibniz Institute for the \\ Social Sciences, Cologne, Germany \\ Full list of author information is \\ available at the end of the article
}

\begin{abstract}
Swarm intelligence is the collective behavior emerging in systems with locally interacting components. Because of their self-organization capabilities, swarm-based systems show essential properties for handling real-world problems, such as robustness, scalability, and flexibility. Yet, we fail to understand why swarm-based algorithms work well, and neither can we compare the various approaches in the literature. The absence of a common framework capable of characterizing these several swarm-based algorithms, transcending their particularities, has led to a stream of publications inspired by different aspects of nature without a systematic comparison over existing approaches. Here we address this gap by introducing a network-based framework - the swarm interaction network - to examine computational swarm-based systems via the optics of the social dynamics. We investigate the structure of social interaction in four swarm-based algorithms, showing that our approach enables researchers to study distinct algorithms from a common viewpoint. We also provide an in-depth case study of the Particle Swarm Optimization, revealing that different communication schemes tune the social interaction in the swarm, controlling the swarm search mode. With the swarm interaction network, researchers can study swarm algorithms as systems, removing the algorithm particularities from the analyses while focusing on the structure of the swarm social interaction.
\end{abstract}

\section{Introduction}

Swarm intelligence refers to the global order that emerges from simple social components interacting among themselves (Bonabeau et al. 1999; Kaufmann 1993; Vicsek 2001; Kennedy and Eberhart 2001; Engelbrecht 2006). In the past three decades, swarm intelligence has inspired many algorithmic models (i.e., computational swarm intelligence), allowing us to understand social phenomena and to solve real-world problems (Engelbrecht 2006). The field of computational intelligence has witnessed the development of various swarm-based techniques that share the principle of social interaction while having different natural inspirations such as ants (Dorigo and Di Caro 1999), fishes (Bastos-Filho et al. 2008), fireflies (Yang 2009), birds (Kennedy and Eberhart 1995), cats (Chu et al. 2006), to name a few. Though researchers have studied such techniques in detail, the absence of general approaches for assessing these systems prevents us from

(c) The Author(s). 2020 Open Access This article is licensed under a Creative Commons Attribution 4.0 International License, which permits use, sharing, adaptation, distribution and reproduction in any medium or format, as long as you give appropriate credit to the original author(s) and the source, provide a link to the Creative Commons licence, and indicate if changes were made. The images or other third party material in this article are included in the article's Creative Commons licence, unless indicated otherwise in a credit line to the material. If material is not included in the article's Creative Commons licence and your intended use is not permitted by statutory regulation or exceeds the permitted use, you will need to obtain permission directly from the copyright holder. To view a copy of this licence, visit http://creativecommons.org/licenses/by/4.0/. 
uncovering what makes them intelligent and understanding the differences between techniques beyond their inspirations.

Much research has been devoted to understanding and improving these bio-inspired algorithms (Kennedy and Eberhart 2001; Engelbrecht 2006; 2007). In the literature, researchers often examine the techniques from the perspective of their natural inspirations. For instance, in some flocking models that mimic bird flocks, the velocities of individuals are usually used to understand the system behavior (Engelbrecht 2007). In these systems, both the lack or excess of spatial coordination among individuals generally leads to poor performance in solving problems. In the case of foraging-based models inspired by ant colonies, many studies attempt to understand the performance of these models by examining the pheromone that agents deposit on the environment (Dorigo and Stützle 2004). This usual approach of analyzing models via their inspiration has helped to improve algorithms by building new procedures (Sun et al. 2004; Dong and Zhou 2017).

These analyses, however, are confined to specific niches that have their metaphor (e.g., ants following pheromone, birds searching for food, fireflies trying to synchronize) and jargon (e.g., pheromone, velocity, fish weight). The wide variety of natural inspirations makes it challenging to find interchangeable concepts between swarm intelligence techniques (Sörensen 2013). The absence of niche-free analyses restricts the findings of a model to its own narrowed sub-field. Such myopia leads us to miss the underlying mechanisms driving a system to the undesired states that new techniques (or procedures) endlessly try to avoid. In this scenario, we need agnostic quantitative approaches to analyze computational swarm intelligence in a general manner and thus provide the means to understand and improve algorithms in whatever niche.

The field lacks general methodologies to analyze swarms because of the absence of a generic framework to examine their main similarity: the social interaction (see Fig. 1). Indeed, the concept of social interaction is fundamental in swarm intelligence; it refers to the exchange of information through diverse mechanisms (Bonabeau et al. 1999; Kennedy and Eberhart 2001). In this definition, social interaction is not only the mere exchange of information between peers but also have the potential to change individuals (Kennedy and Eberhart 2001). The sophisticated behavior emerging from social interaction enables the system to adjust itself to solve problems (Kennedy and Eberhart 2001). In swarm intelligence techniques, individuals process information and interact locally among themselves, spreading knowledge within the swarm, which results in the emergent system ability. In this sense, examining the social mechanisms is fundamental to understand intelligence in these systems. This general perspective also helps us to assess swarms with different natural inspirations. Instead of relying on the complete understanding of the micro-level

\section{Swarm Intelligence}

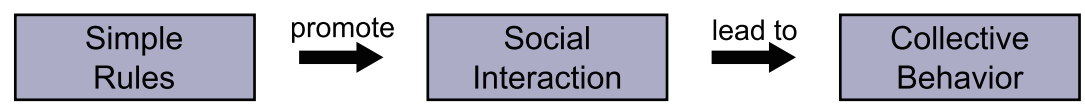

Fig. 1 The swarm social interaction at the meso level is still overlooked by researches who often devote considerable efforts to understand how changing the simple rules at the micro level (e.g., procedures, equations) directly affects the collective behavior of the system at the macro level. In fact, these micro-level rules create the conditions to social interaction at the meso level which in turn enables the necessary swarm dynamics to solve complex problems at the macro level 
properties (e.g., velocity, pheromone, weight), we can assess the swarm via the structure and dynamics of the social interaction (Bonabeau et al. 1999).

Notably, the field of Network Science has shown that every complex system can be represented as a network encoding the interactions between the components of the system and that the understanding of the structure of this network is sine qua non for learning the behavior of the system itself (Barabási 2012). Network Science advocates that the understanding of complex systems can be reached by observing the structure and dynamics of their underlying networks (Strogatz 2001; Barabási 2012). Though the idea of using networks as frameworks for understanding complex phenomena dates back to Moreno's use of sociograms in the 1940s (Moreno 1946), it has been popularized by two seminal papers from Watts and Strogatz (1998), and Barabási and Albert (1999) in the late 1990s. Recent works in the field have demonstrated that even small variations in fundamental structural properties, such as degree distribution, can significantly influence the behavior of the system described by the network.

Here we propose a network-based framework-the swarm interaction network-for the general assessment and comparison of swarm intelligence techniques. With this framework, we study swarm algorithms as systems, removing the niche-specific particularities from the analyses. The swarm interaction network differs from other more general network science frameworks, such as temporal networks (Holme and Saramäki 2012), in the focus on making swarm algorithms more transparent. In the following sections, we start by describing the importance of understanding swarm-based algorithms, and by explaining the definition of the interaction network. We show how the interaction network can be defined for four well-known swarm-based algorithms from two distinct categories, as proposed by Mamei et al. (2006), namely flocking and foraging. Then, we demonstrate a complete case study using the concept of flocking and show the relationship between the interaction network and the swarm search mode.

\section{Understanding swarm systems}

In the field of Computational Swarm Intelligence, scholars often analyze algorithms via their performance on given problems. In many cases, innovation means the development of novel algorithms that are capable of achieving improved results on a set of benchmark functions. These improvements, however, tend to arise without much explanation. Researchers also often use jargon in both the justification for novel algorithms and the description of their improvements (Sörensen 2013). This black-box approach not only hinders the interpretability of results but also sidetracks us from the underlying mechanisms driving the swarm intelligence in these systems. The case occurs because of the lack of a unifying view of swarm-based algorithms. Though some efforts have been made to understand swarm systems from a general perspective, they tend to be qualitative in nature.

The general perspective for swarm-based systems proposed by Mamei et al. is that of a system processing information (Mamei et al. 2006). From this perspective, the way a swarm handles information defines its underlying self-organization mechanism. We can describe a system using three aspects of information: (i) the definition of information, (ii) how individuals use information, and (iii) how information flows within the system (see Fig. 2). This approach classifies swarm systems but fails to examine them quantitatively. 


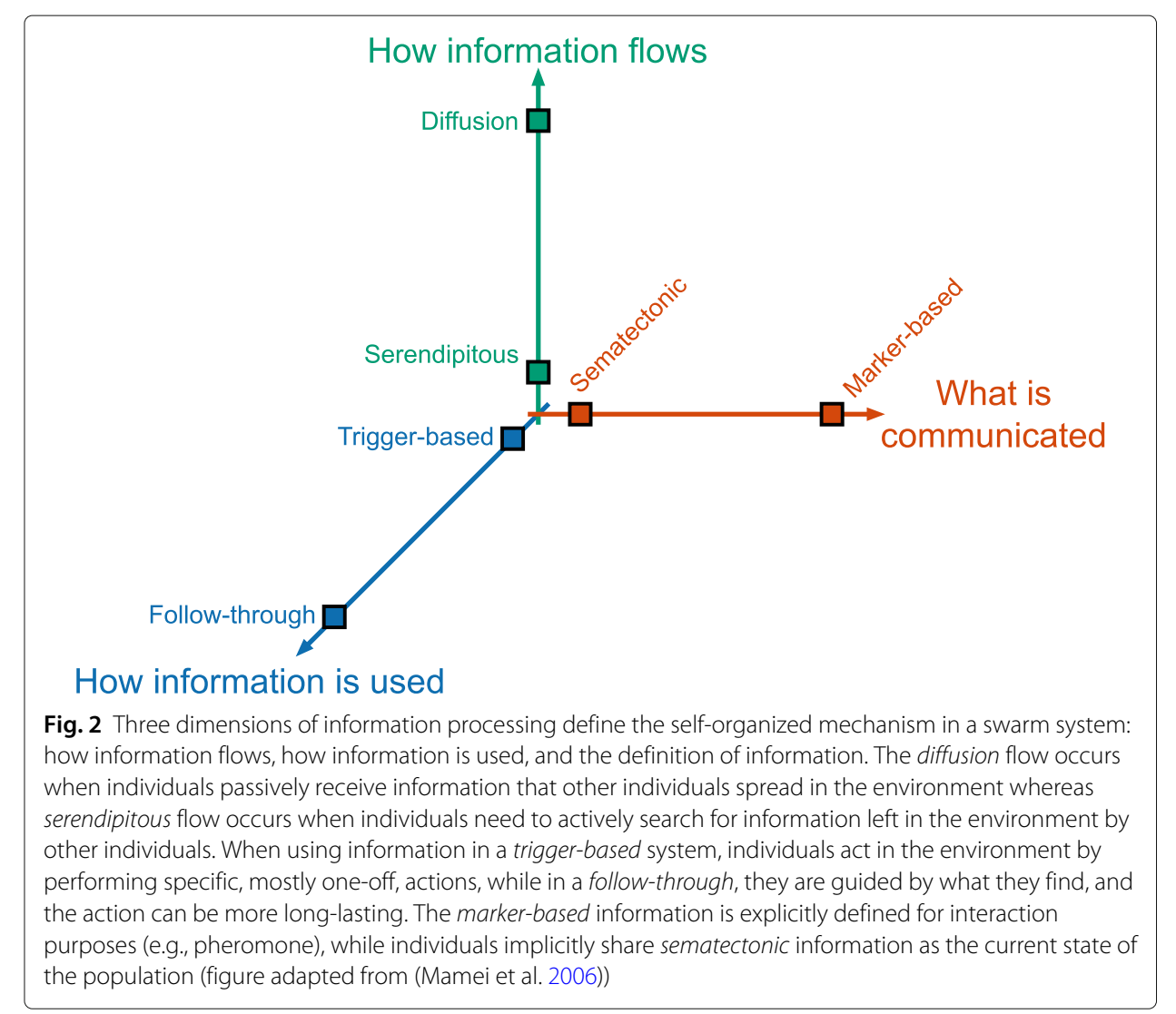

In fact, the literature has various approaches to classify swarm systems (Parpinelli and Lopes 2011; Duan and Luo 2015; Chu et al. 2018) and metaheuristics in general (Gendreau and Potvin 2005; Fernandez-Marquez et al. 2012; Fong et al. 2015). These efforts are essential to organize the field. They are the necessary initial steps to understand current and new algorithms. Still, the absence of quantitative approaches prevents us from characterizing the particularities of methods and quantifying their differences.

In some cases, researchers measure the swarm diversity to understand swarm-based techniques (Chu et al. 2018). This diversity is often the diversity of the candidate solutions when solving a given problem (Engelbrecht 2007; Dorigo and Stützle 2004; Krink et al. 2002; Shi and Eberhart 2008; Olorunda and Engelbrecht 2008; Shi and Eberhart 2009). With such an approach, however, we focus on the outcome of the swarm dynamics, neglecting the underlying mechanism leading to these dynamics. We lack a framework enabling us to examine the system from an intermediate perspective.

\section{The social interaction in swarm systems}

The dynamics of swarm-based systems depend on social interaction. The system lacks coordination without enough interaction among the individuals and loses adaptability with the excess (Chaté and Muñoz 2014). In such systems, the local rules promote or undermine the level of interaction within the swarm (Fig. 1). In this sense, the swarm social interaction is halfway between the micro and macro behavior of the system. The network emerging from these complex interactions is a natural universal meso-level perspective of swarms. 
Previous research has used the network paradigm to examine emergent behavior in social animals and their underlying mechanisms (Fewell 2003; Lusseau 2003; StrandburgPeshkin et al. 2013; Rosenthal et al. 2015). Many works have also shown that collective behavior can emerge from simple rules (Fehr and Gächter 2000; Xia et al. 2012; Meloni et al. 2017). In the computational intelligence field, some developments have taken advantage of networks (Giacobini et al. 2006; Payne and Eppstein 2009; Godoy and von Zuben 2009; Dorronsoro and Bouvry 2012; Oliveira et al. 2013; Liu et al. 2014; Metlicka and Davendra 2015). They have been used to understand swarm systems (Whitacre et al. 2008; Huepe et al. 2011) and their respective collective behaviors such as flocking (Oliveira et al. 2013, 2014, 2015, 2016, 2017; Janostik et al. 2016; Pluhacek et al. 2016; Wen-Bo et al. 2016) and foraging (Metlicka and Davendra 2015; Kromer et al. 2015). In this regard, Oliveira et al. proposed one of the first approaches to examine interactions within the swarm in the Particle Swarm Optimization (Oliveira et al. 2013). Yet, these preliminary efforts have focused on specific techniques, missing the fact that social interaction is the characteristic feature driving swarm intelligence.

In this work, we argue that the social dynamics in swarm-based algorithms should be more analyzed and explored to provide insights into the network behind the rules and inspirations, which may lead to a possible meta-classification of the systems-a metaclassification based on the system dynamics instead of the natural inspiration of the system. In the following section, we define the interaction network and use the categorization proposed by Mamei et al. to elaborate on the plausibility of describing and employing the interaction network to assess models of swarm intelligence inspired by different mechanisms of self-organization. Through various mechanisms, an interaction network can be built to characterize the system over a shared space: the swarm interaction space. In this space, the swarm interaction network becomes a general framework that allows for the unified assessment of swarm intelligence models with distinct inspirations.

\section{The network of social interaction}

We propose to examine the social interaction within a swarm as a way to assess the behavior of swarm intelligence systems. Here we develop the concept of swarm interaction network to represent the interdependencies of the actions of the individuals. For a given swarm system, the swarm interaction network I consists of nodes that represent its individuals and edges $\mathbf{I}_{i j}$ that indicate the extent to which individual $i$ influences the action of the individual $j$. As the swarm social interaction is dynamic and so the swarm, we use $\mathbf{I}(t)$ to describe the influence that individuals exert on each other at time $t$.

The interaction network is a representation of the swarm and the result of the rules that define the swarm system. Though these rules are bio-inspired, the network $\mathbf{I}$ belongs to the swarm interaction space $\mathcal{I}$ (see Fig. 3). This is an agnostic space exempt from the particularities of the swarm algorithm or problems being solved by the algorithm. Note that both the algorithm (i.e., rules) and problem modify the social dynamics within the system and have an impact on I. Yet, when we look at algorithms from this general framework, we have the potential to assess different algorithms that are, at their surface, completely distinct (i.e., inspired by distinct natural phenomena).

The network structure-at both global- and individual-levels-enables us to analyze different aspects of the swarm and aspects across different swarm intelligence approaches. For instance, Fig. 4 depicts a conceptual interaction network for swarm 


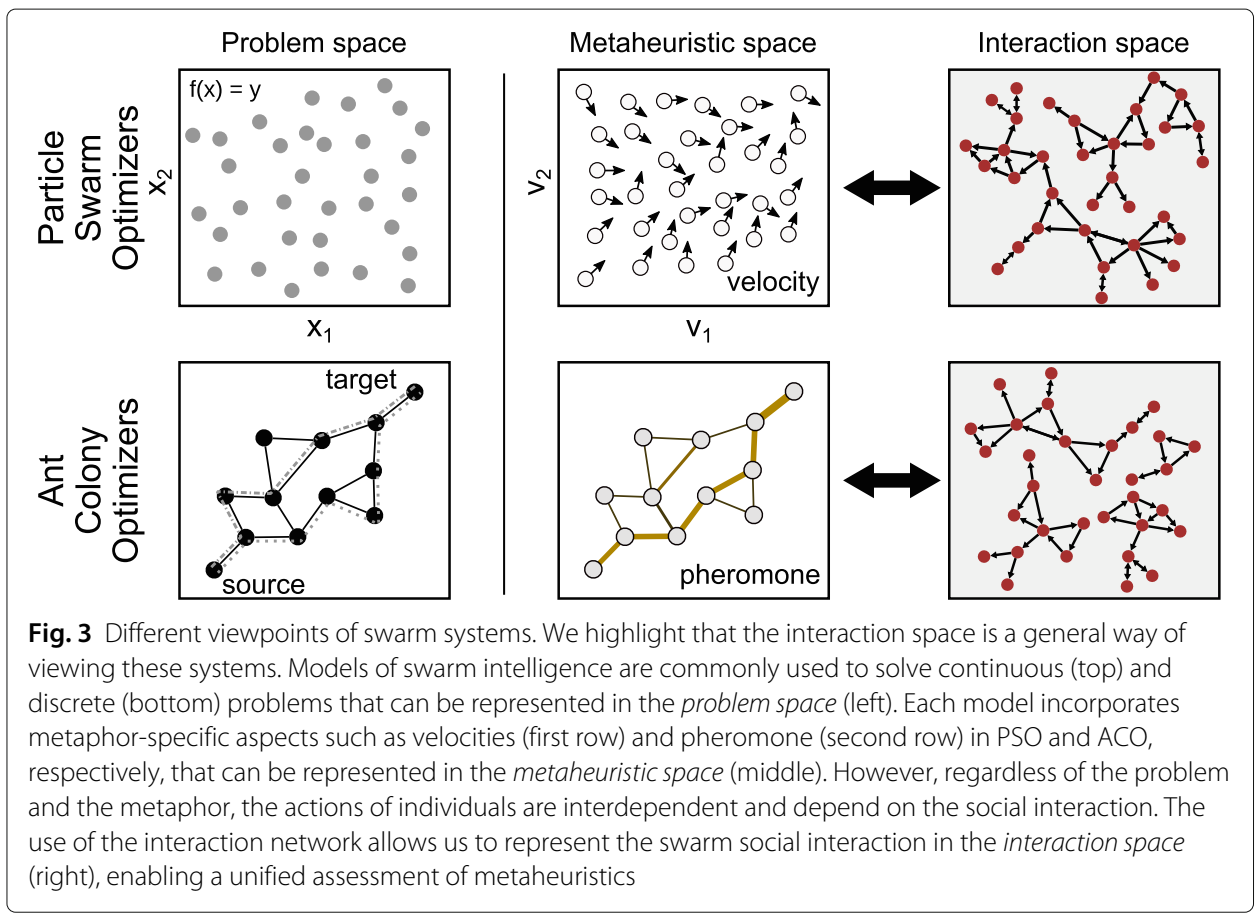

systems. At the individual level, the network positions occupied by individuals indicate the types of interdependencies that were created by the swarm and the influence individuals may exert on one another. The individuals with a high degree centrality (e.g., individuals 1 and 12) typically exert stronger influence when compared to other individuals. Similarly, individuals that connect different groups (e.g., individual 9) act as bridges between groups of individuals and control the cascade of influence between subnetworks. Thus, some individuals in a swarm system can develop important roles as bridges and hubs. Lastly, at the global level, the interaction network indicates the extent of local and global exploration by providing the relationship between natural niches formed by individuals (e.g., green and blue sub-networks).

To analyze a swarm using the interaction network, we need to learn the rules and mechanisms that allow individuals to influence the action of each other within the system. We use the dimensions described in Fig. 2 to guide our understanding of algorithms and thus to define their networks. For a given algorithm, we have to identify how an individual

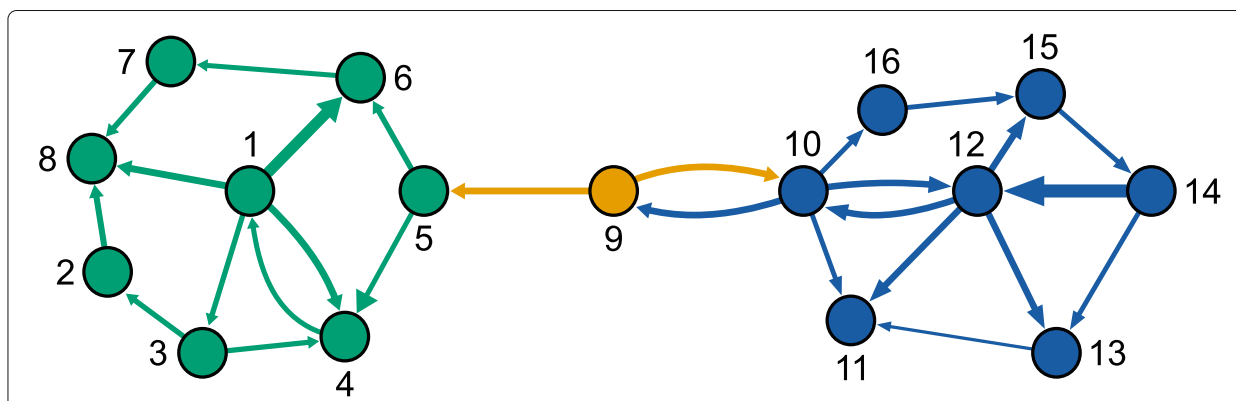

Fig. 4 A general illustration of an interaction network for swarm systems where each node is an individual in the population, and each link represents the direction and the extent of the individuals' influence. Each color depicts a distinct sub-network to which members are highly integrated and tightly connected when compared to nodes outside the sub-network 
uses information after it received information from other individuals. We need first to characterize what information is in the system then describe how information exchange influences individuals. Table 1 describes how the interaction network can be constructed for each category proposed by Mamei et al. (2006).

In the following subsection, we use the interaction network to compare four swarmbased algorithms from two distinct categories, namely flocking and foraging. In this brief analysis, we show that simple definitions of the interaction network enable us to recognize differences and similarities among these algorithms. Then, we provide a more detailed case study of PSO in which we study the dynamics of the swarm at the meso level of the social interaction.

\section{Modeling the swarm interaction network}

With the swarm interaction network, we examine the social interaction in four bioinspired optimization algorithms: the Artificial Bee Colony (ABC) (Karaboga 2005), the Ant Colony Optimization (ACO) (Dorigo and Stützle 2004), the Particle Swarm Optimization (PSO) (Kennedy and Eberhart 1995), and the Fish School Search (FSS) (Bastos-Filho et al. 2008). These algorithms have distinct inspirations and are from separate categories, but they share the same concept of social interaction. In this section, we avoid technicalities and save them to the next section, where we delve into a specific case study. Instead, here we discuss these algorithms from a high-level perspective, focusing on the social interaction. First, we describe how agents in the system influence each other; then, we build a network based on this description.

The core of these algorithms is that successful individuals are more likely to influence the population of the system. Though the definition of success depends on the algorithm, it generally relates to the quality of a solution. The agents navigate through a metaheuristic space relying on the information from other agents and the environment. We describe the interdependency in the actions (e.g., swimming, flying) of the individuals using the interaction network. For each algorithm, we build the network $\mathbf{I}(t)$ in that each link represents this interdependency between two individuals (i.e., nodes) at iteration $t$.

In the case of the PSO algorithm, particles move towards the best particle (i.e., the most successful one) in their neighborhoods at each iteration $t$. To describe this influence with the interaction network $\mathbf{I}(t)$, we connect each agent $i$ to its best neighbor $j$ at each iteration $t$. Note that particles move using information from only one individual in their neighborhood.

Fishes in the FSS algorithm use information from all individuals in the fish school. Each fish contributes to the movement of the fish school based on its current displacement and its previous success. We describe this interdependency with a weighted network. We build a network in which the weight represents the proportional contribution of the individual $i$ to the movement of the individual $j$ at the iteration $t$.

Note that both PSO and FSS are deterministic with regards to the interaction among individuals. The success of the agents determines their interaction with other agents. In the case of the $\mathrm{ABC}$ algorithm, social interaction takes place only probabilistically.

In the $\mathrm{ABC}$ algorithm, bees fly using information from bees that are selected based on a uniform distribution and a roulette wheel. The former enables an agent to influence any other agent regardless of success, whereas the latter tends to drive agents to interact with successful ones. We build the network $\mathbf{I}(t)$ based on this selection process. Precisely, we 


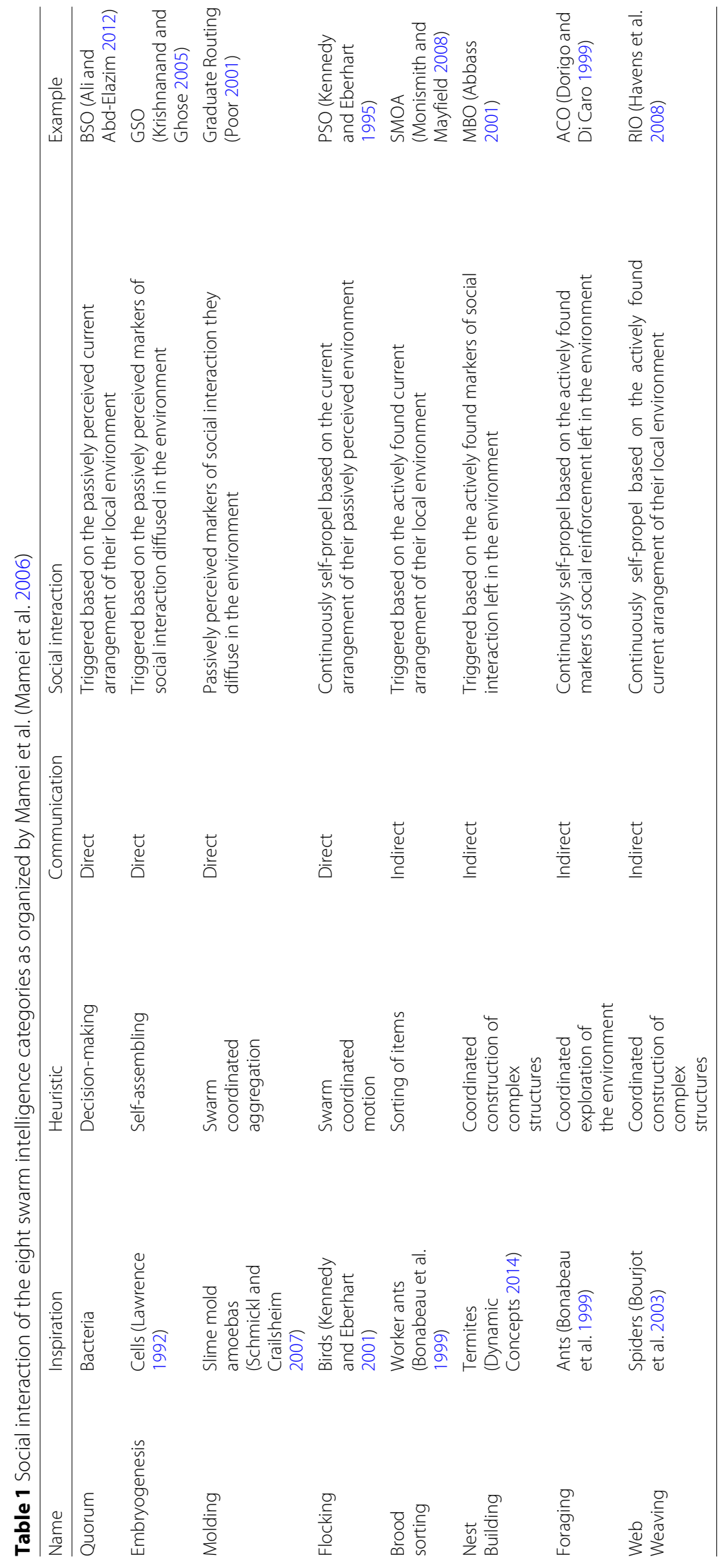


create an edge (or increment the weight) between the agent $i$ to the agent $j$ every time the agent $i$ considered the position of agent $j$ to move at the iteration $t$.

In the algorithms described above, individuals can communicate directly with each other. Notably, the Ant Colony Optimization algorithm uses the concept of stigmergy in which the agents communicate only indirectly via pheromones. In ACO, each ant moves across the environment (a graph) following higher concentrations of pheromone and depositing pheromone at visited edges accordingly. In this case, we build a weighted interaction network by keeping track of the amount of pheromone deposited by each ant $i$ at each edge. The influence $i$ exerts on $j$ is the cumulative amount of pheromones left by $i$ at the edges visited by $j$.

With these network definitions, we run an implementation of each algorithm and examine the social interaction in these systems. For each case, we analyze the swarm accounting for the whole execution, so we sum up matrices over time as follows: $\mathbf{I}=$ $\sum_{t} \mathbf{I}(t)$. These matrices (Fig. 5a) and the node strength distributions (Fig. 5b) can help us study the patterns of social interactions among these algorithms. The right skewness in the node strength distribution, for instance, indicates how likely is the emergence of highly influential individuals (i.e., hubs) which are more likely in the PSO and FSS algorithms and less likely in the ABC algorithm. The likelihood of hubs in the interaction network of these algorithms can regulate the extent of exploration and exploitation as the presence of hubs within the swarm can boost the information sharing in the system that accelerate its pace of convergence. The extent of exploration and exploitation, however, depends on the problem. In unimodal problems, for instance, the presence of hubs can

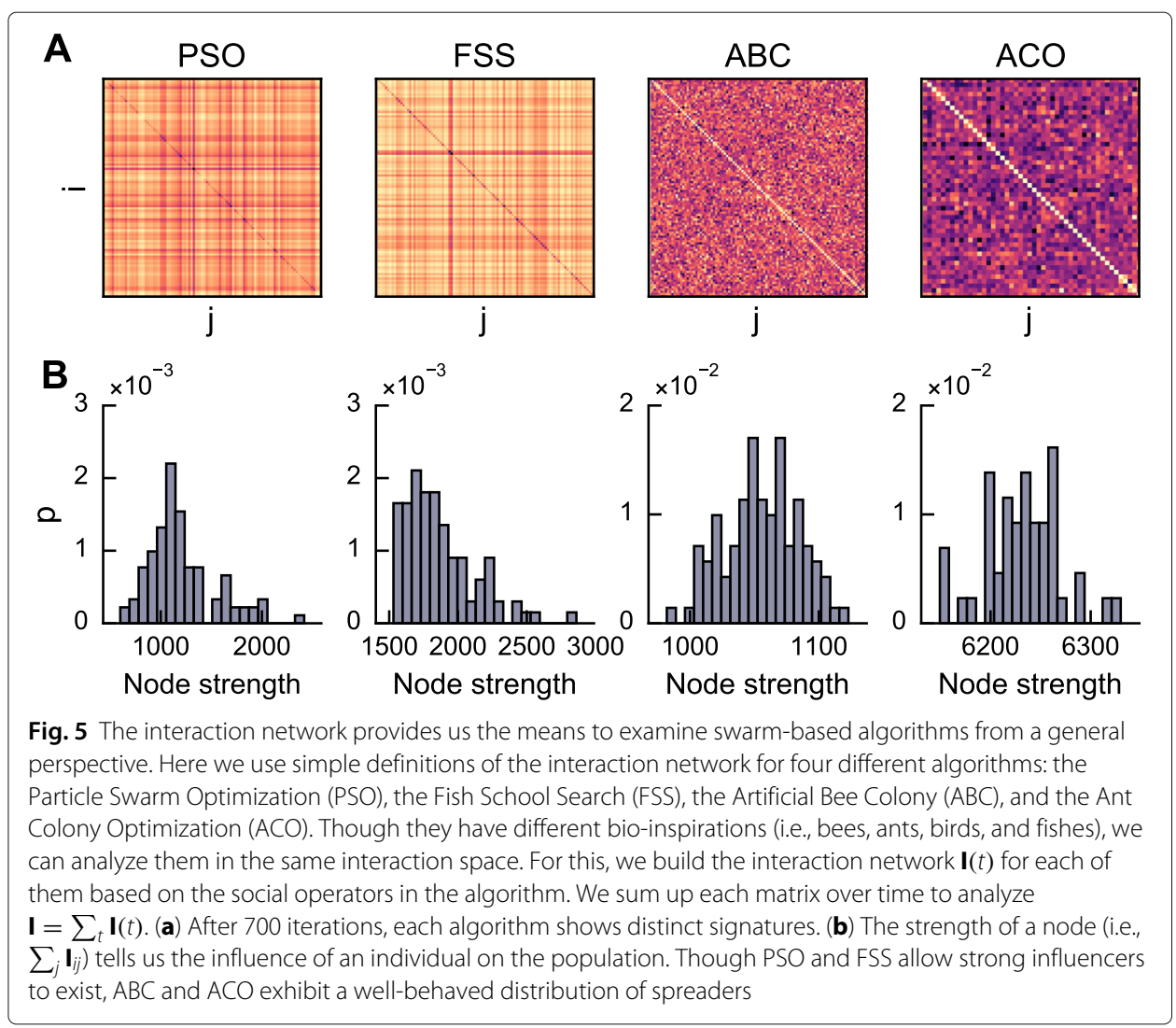


be positive, and a faster convergence can quickly attract the swarm to optimal states. In some multimodal problems, however, the presence of hubs can be negative, and a faster convergence may attract the swarm to suboptimal states. Thus, the interaction network provides us the means to study these peculiarities in swarm intelligence algorithms from a general perspective-a necessary step towards understanding swarm intelligence. The framework also creates an opportunity to study meso-level dynamics, as we show in the next section.

\section{In-depth case study: examining the particle swarm optimization algorithm}

In this section, we present how to analyze the meso-level dynamics of a swarm-based algorithm using the interaction network. We select the PSO algorithm because of its simplicity and extensive use in several applications.

Particle Swarm Optimization (PSO) is a population-based optimization method that relies on the interactions of individuals sharing the best positions they found during the search process (Kennedy and Eberhart 1995). The method-inspired by the social behavior in flocks of birds-consists of a population of simple reactive agents (particles) that explore the search space by locally perceiving the environment and interacting among themselves to improve their solutions.

In the standard definition of the PSO, each particle $i$ consists of four vectors in a $d$ dimensional search space: its current position $\vec{x}_{i}(t)$, its best position found so far $\vec{p}_{i}(t)$, its velocity $\vec{v}_{i}(t)$, and the best position found by its neighbors $\vec{n}_{i}(t)$ (Bratton and Kennedy 2007). The position of each particle represents a candidate solution to a $d$-dimensional continuous optimization problem, and the swarm moves through the problem search space seeking better solutions. To enable this capability, all particles change their positions, at each iteration $t$, according to their velocities $\vec{v}_{i}(t)$ which are updated based on the personal best position $\vec{p}_{i}(t)$ and the social best position $\vec{n}_{i}(t)$. Researchers use different ways to update the position of the particles, but the update equation generally aims to maintain the coherence of the particles through an inertia term and adjust the trajectory using cognitive and social information. In our study, we use the so-called constricted PSO (Clerc and Kennedy 2002).

The particles in the swarm only interact with a subset of the swarm. The swarm topology defines the infrastructure through which particles communicate and thus enables the particles to retrieve information from other particles (i.e., their neighbors). At each iteration $t$, each particle $i$ seeks for its best neighbor $n_{i}(t)$ in its neighborhood (i.e., the one with the best solution so far). The topology influences the social interaction within the swarm and has been shown to impact the swarm performance (Mendes 2004; Bratton and Kennedy 2007). Clerc proposed a somewhat different definition of swarm topologythe so-called graph of influence-which explicitly includes the social information and presents directed edges (Clerc 2010). Regardless of definition, however, the swarm topology only refers to the structure for the potential exchange of information and neglects effective interaction among particles.

In the particle-swarm context, exploration and exploitation refer to the ability of individuals to broadly explore the whole search space or focus on a particular area (Kennedy and Eberhart 2001). An exploration-exploitation imbalance often leads to a poorly explored search space. To better understand this imbalance, researchers study the diversity and the dynamics of the swarm. The literature often focuses on the spatial diversity 
(Shi and Eberhart 2008; Olorunda and Engelbrecht 2008; Shi and Eberhart 2009), analyzing the outcomes of social interaction such as the positions or velocities of the particles in the search space. These approaches have succeeded in developing novel mechanisms to improve the performance of the algorithm. Yet, with these approaches, we fail to understand the underlying social interaction driving the swarms to undesired states (e.g., lack of diversity, premature convergence) that new mechanisms try to avoid.

Still, a few works have attempted to analyze the particles' interactions in order to examine the swarm behavior. Some of these efforts analyzed the impact of the infrastructure of the swarm communication on the swarm performance (Mendes et al. 2004; Mendes 2004; Wen-Bo et al. 2016). Though these studies neglected the actual interactions between particles, they showed that bounding social interaction influences the swarm behavior. Oliveira et al. proposed to examine the actual interactions among particles in order to assess the swarm (Oliveira et al. 2013). They proposed the analysis of the swarm using a network in which the nodes (particles) are connected if they share information in a given iteration and later extended the concept to capture historical information (Oliveira et al. 2014, 2015, 2017). Later on, Pluhacek et al. provided visualizations of the interactions in the swarm (Pluhacek et al. 2016).

In the next subsections, we define the interaction network I to assess the swarm using the methods developed by Oliveira et al. (2016). With this definition, we can uncover the interplay of swarm dynamics, swarm performance, and social interaction.

\section{A network for the particle swarm optimization}

To examine a swarm system from the meso-level perspective of the swarm social interaction, we need to build the network to capture the structure and dynamics of the social influence exerted among individuals. In the case of the PSO algorithm, social interaction occurs when a particle $i$ updates its position based on the position of a particle $j$. This happens when $j$ is the best neighbor of $i$ at a given iteration; that is, $n_{i}(t)=j$.

Here we use a simple (yet powerful) definition of interaction network $\mathbf{I}(t)$ in which the weight of an edge $(i, j)$ is the number of times the particle $i$ was the best neighbor of the particle $j$ or vice-versa until the iteration $t$ (Oliveira et al. 2014). We use a time window $t_{w}$ to control the recency of the analysis, thus the interaction network at iteration $t$ with window $t_{w}$ is defined as the following:

$$
\mathbf{I}_{i j}(t)=\sum_{t^{\prime}=t-t_{w}+1}^{t}\left[\delta_{i, n_{j}\left(t^{\prime}\right)}+\delta_{j, n_{i}\left(t^{\prime}\right)}\right]
$$

with $t \geq t_{w} \geq 1$ and where $\delta_{i, j}$ is Kronecker delta. In this definition, nodes (i.e., particles) are connected by an edge with weight equals to the number of times two particles shared information in at most $t_{w}$ iterations before the iteration $t$ (Oliveira et al. 2014). The time window $t_{w}$ tunes the frequency-recency balance in the analysis. High $t_{w}$ makes the network dominated by most frequent interactions; low $t_{w}$ only includes most recent interactions, and when $t_{w}=1$ we have instantaneous interactions.

Note that the definition of an interaction network for a swarm system depends on the rules that promote social interaction in the system. Here we pinpointed that, in PSO, social interaction between $i$ and $j$ occurs when the particle $i$ updates its velocity $\vec{v}_{i}$ using the position of a particle $j$. This definition of $\mathbf{I}$ is a simple one that includes only the occurrence of social interaction between particles. More complex definitions may include edge 
direction or other aspects of the algorithm, such as the social constant $c_{2}$ or the realizations of random numbers. Nevertheless, with this simple definition, we can already better understand the swarm (Oliveira et al. 2013; 2014; 2015; Oliveira et al. 2016; Oliveira et al. 2017). Other swarm systems, however, have different rules and distinct forms of social interaction.

\section{Examining the social interaction with I}

The formation of structures in the interaction network arises from the way information flows within the swarm, which, in turn, alters the dynamics of the swarm. The existence of well-connected nodes in $\mathbf{I}$ indicates frequent information flows in the swarm. The constant interaction among specific individuals leads to their respective nodes in the interaction network to be clustered. To capture these clusters, we can gradually remove the edges of I according to their weight; the components that emerge during this network destruction represent the information flows within the swarm (see Fig. 6).

Note that the pace at which these components appear relates to the swarm dynamics. A slow increase suggests an exploration search mode in which individuals share information among distinct groups and thus create social interaction with various levels of tie strength. A rapid growth suggests, however, an exploitation search mode in which individuals interact with a few same sources and thus create a center of information with similar levels of tie strength.

With the definition in Eq. (1), we can now examine the search mode in the PSO algorithm. For instance, we analyze I of swarms using different topology parameters-that are known to lead the swarm to behave differently-while solving the same problem. As shown in Fig. 7a, with the global topology, the particle swarm presents exploitation behavior, whereas the ring topology leads the system to explore different information sources. Note that this analysis differs from the typical analysis of the relationship between fitness and topology structure (Mendes et al. 2004; Mendes 2004; Engelbrecht 2013). Here we focus on the way particles interact during the swarm search when using different structures: the communication topology affects the diversity of the social interaction in the swarm.

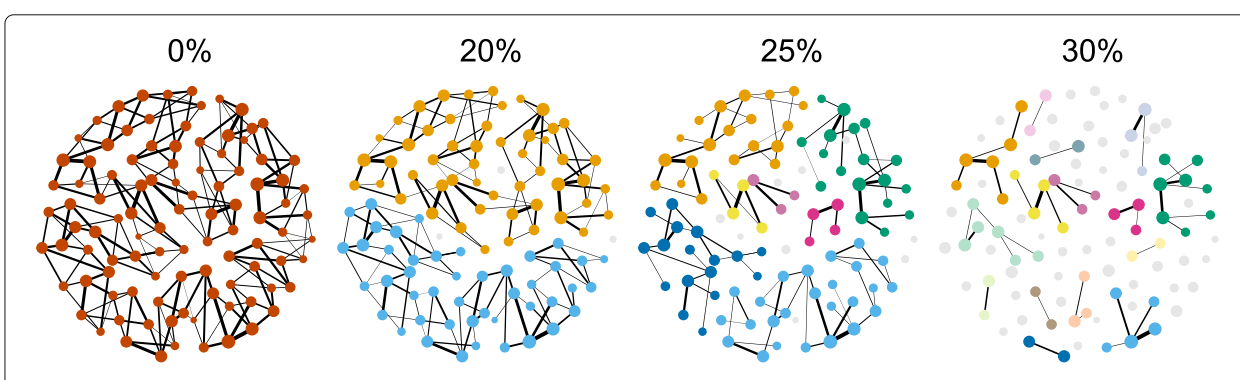

Fig. 6 The pace at which components emerge while edges are gradually removed from $\mathbf{I}$ is associated with the search mode of the swarm. An exploration mode is characterized by a slow increase in the number of components due to the different information flows present in the swarm. The network, however, is rapidly destroyed in a swarm that depends only on a small set of individuals, a behavior related to an exploitation search mode. In PSO, the weighted interaction network of a run with the swarm using a von Neumann topology has edges removed based on their weight: below $20 \%$ of the highest possible weight, $25 \%$ and $30 \%$. The colors represent components with more than one node. In this process, edges with the lowest weights are removed first 


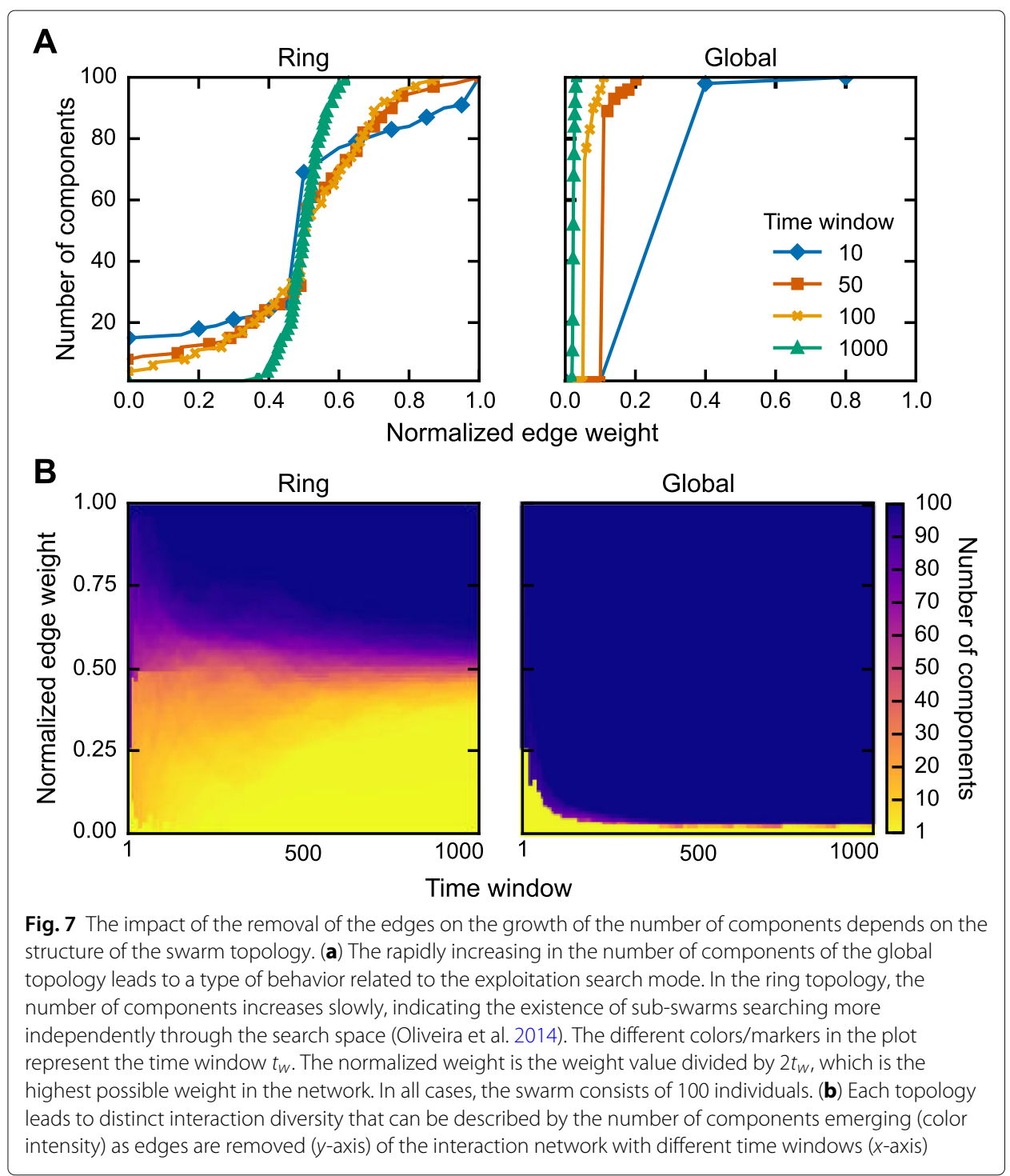

To investigate the swarm ability to maintain different frequent information flows, we can analyze the network destruction while varying $t_{w}$ to include frequency and recency in the analysis of the flows. Figure $7 \mathrm{~b}$ depicts the number of components that emerge when edges are removed from $\mathbf{I}$ with increasing time windows. The interaction network of a particle swarm with global topology seems to be destroyed at the same pace in both perspectives of frequency (i.e., high $t_{w}$ ) and recency (i.e., low $t_{w}$ ). The interactions of the particles within this topology promote a lack of diversity in the information flows in short and long terms.

This diversity regards to the ability of the swarm to have a diverse flow of informationa perspective different from spatial diversity in which $d$-dimensional properties of particles are compared to particular definitions of swarm center (Shi and Eberhart 2008). Note that the lack of diversity in the information flow can decrease the spatial diversity in a swarm. The absence of multiple information flows leads to particles retrieving information from a few sources and drives particles to move towards the same region of the search space; lack of interaction diversity pushes individuals to the same direction. 
To quantify interaction diversity, we measure the destruction pace of interaction networks with different time windows. For a given time window $t_{w}$, the area under the destruction curve $A_{t_{w}}$ can be seen as a measure of diversity in the information flow. High values of $A_{t_{w}}$ indicate fast destruction, whereas low values imply slow destruction. Hence, we can define the interaction diversity ID (previously called communication diversity (Oliveira et al. 2016)) as the mean diversity over a set of time windows $T$, as the following:

$$
\mathrm{ID}(t)=1-\frac{1}{|S||T|} \sum_{t_{w}^{\prime} \in T} A_{t_{w}=t_{w}^{\prime}}(t),
$$

where $|S|$ is the number of particles in the swarm. Thus, swarms exhibiting high ID (i.e., low values for $A_{t_{w}}$ ) have the ability to have diverse information flows, while low values for ID imply swarms with only few information flows (i.e., high value for $A_{t_{w}}$ ). The ideal set $T$ would be one taking into account all time windows (i.e., from $t_{w}=1$ until $t_{w}=t$ ). This procedure, however, can be computationally expensive because of the vast number of possible time windows; instead, we use a subset of time windows.

\section{Experimental design}

To investigate the extent to which the interaction diversity assesses the swarm at each iteration, we systematically examined the swarm using different topologies that lead the swarm to behave differently. In this study, we use $k$-regular networks (i.e., graphs in which nodes have $k$ links) as the swarm topologies. The $k$-regular is a simple network model that enable us to create topologies with intermediate connectivity between ring $(k=2)$ and global $(k=N)$, the two most used swarm topologies. To create a diverse set of topologies, we employ different connected $k$-regular graphs as the swarm communication topology with $k$ ranging from 2 to 100 . Here we consider a distinct group of four benchmark functions $F_{2}, F_{6}, F_{14}$, and $F_{19}$ from the CEC'2010 (Tang et al. 2010) which require different balances of exploration/exploitation (Oliveira et al. 2017). In all experiments, the number of dimensions to 1000 and, when applicable, the degree of non-separability $m$ to 50 ; also, we set the swarm size to 100 particles.

We analyze the relationship between ID and fitness improvement over time; thus we define fitness improvement $f_{\Delta}(t)$ at iteration $t$ as the speed at which the fitness $f_{g}(t)$ of the swarm changes between the two immediate iterations $t$ and $t-1$ as follows: $f_{\Delta}(t)=\frac{f_{g}(t)-f_{g}(t-1)}{f_{g}(t-1)}$, where $f_{g}(t)$ is the global best fitness of the swarm at iteration $t$. To compare the social interaction in similar scenarios in the simulations, we set as stopping criterion either a maximum number of iterations $t_{\max }=10,000$ is reached or the swarm has converged at iteration $t_{s}$. We define that a swarm converged at iteration $t_{s}$ if the global best fitness does not improve, that is, if $f_{\Delta}(t)<10^{-5}$, until iteration $t_{s}+\delta$ with $\delta=500$. In this sense, we ensure that the swarm either converged or reached the maximum number of 10,000 iterations. For each considered swarm topology, we run a PSO implementation 30 times while measuring ID and $f_{\triangle}$ at each iteration in each execution.

\section{Results}

We analyze the impact of the infrastructure of communication (i.e., topology) on the diversity of the information flows within a swarm. We found that $k$-regular topologies promote higher diversity as $k$ decreases when solving the same problem (Fig. 8a). With less connected topologies, swarms exhibit greater interaction diversity than with more 

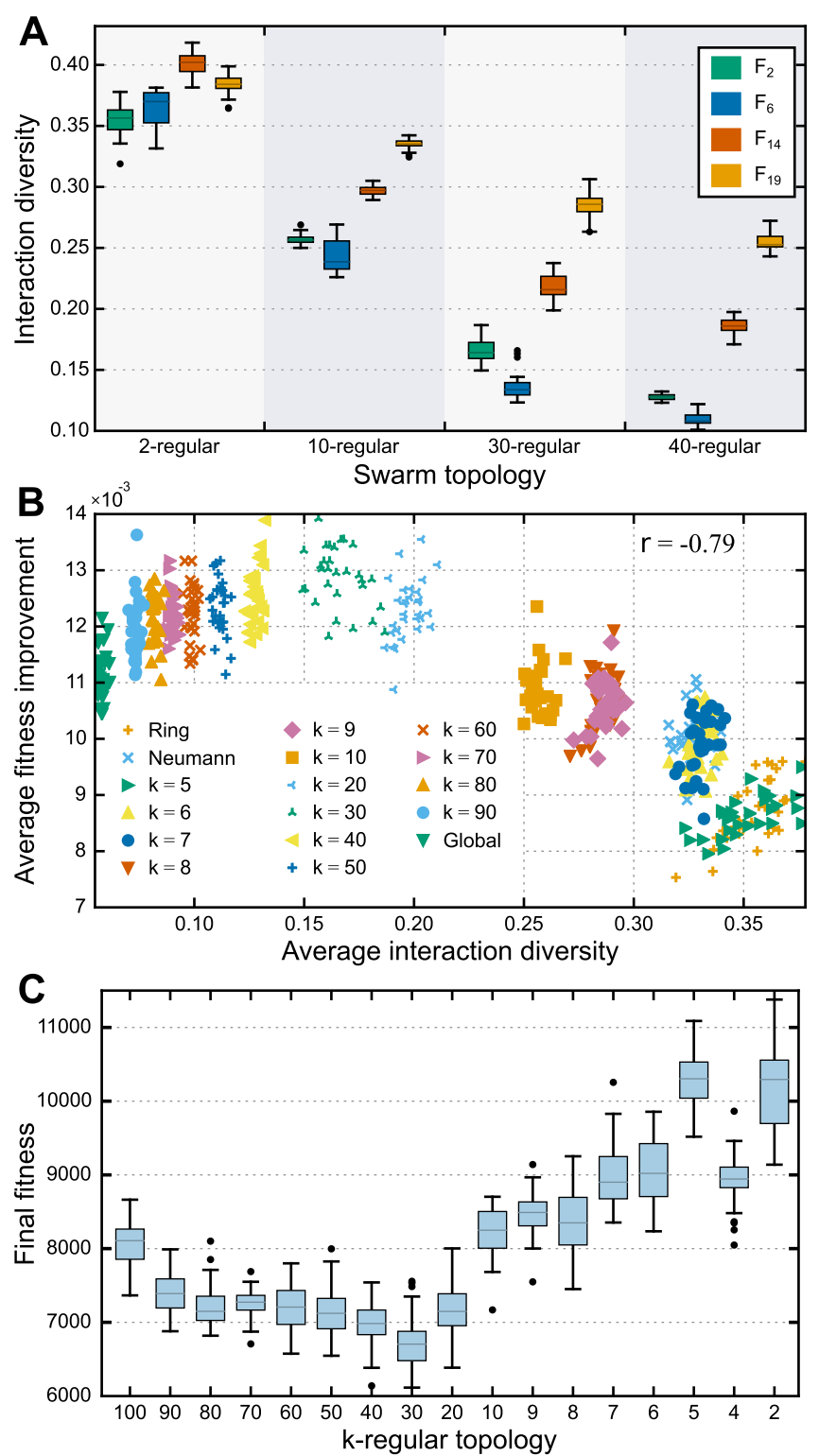

Fig. 8 The interaction diversity, fitness improvement, final fitness, and $k$-topologies are associated in a non-trivial manner. (a) Some benchmark functions appear to consistently present higher interaction diversity when compared to other regardless of the underlying communication topology. In the results for $F_{6}$ function, (b) although the correlation of -0.79 indicates a strong negative linear relationship between the average interaction diversity and the mean fitness improvement, one can easily see that they are associated in a non-monotonic way. (c) Similarly, the final quality of the fitness found by the swarm also presents a non-monotonic behavior regarding $k$-regular topologies and consequently, interaction diversity

connected ones. Given previous studies, this is an expected result: short topological distances lead to fast information flow, which decreases the diversity (Bratton and Kennedy 2007). Our results revealed that the interaction diversity in the swarm depends on the problem; the same topology leads to distinct levels of diversity when optimizing different functions. Though the topology bounds the interactions among particles, the swarm organizes the information flows to optimize a function. 
Indeed, swarm intelligence systems have the capability to self-organize during the optimization process. To assess the relationship between swarm search and interaction diversity, we examine the pace of $f_{\Delta}$ at which a swarm improves and the interaction diversity at each iteration. We find that ID exhibits a non-trivial relationship with $f_{\Delta}$, as seen in Fig. $8 \mathrm{~b}$ for the function $F_{2}$. The average $f_{\Delta}$ increases with the average ID until reaches a maximum pace after which $f_{\Delta}$ decreases with ID. The increase of diversity in the social interaction of the swarm leads to faster swarm pace only until a certain level of diversity; then the swarm starts to slow down-swarm dynamics that impact the overall swarm performance, as seen in Fig. 8c. We also find a non-trivial association between $k$-regular topologies and the best fitness found at the end of the optimization process. From global to 30-regular topologies, the fitness decreases from $8.06 \times 10^{3}$ and improves down to $6.77 \times 10^{3}$, then deteriorates up to $1.01 \times 10^{4}$.

\section{Discussion}

Our results demonstrate the capability of interaction diversity, ID, to explain the behavior of the swarm during the optimization process in the Particle Swarm Optimization technique. ID enables us to identify changes in the way information flows within the swarm regardless of the type of problem and perhaps even variations of PSO (e.g., with our without local search). The leverage capability of the proposed approach brings the possibility to identify imbalances during the search process and to understand the flow of information within the swarm. For example, more than using this approach to select which is the best topology for a particular problem (Engelbrecht 2013), one can propose adaptive mechanisms to adjust the search mode during the search process. Furthermore, note that the Interaction Diversity is a general measure to assess swarm-based systems because it does not consider peculiarities associated with the swarm metaphor. The approach is defined over the structure of the network-the interaction space-which is entirely based on the swarm social interaction. This approach can also help researchers to perform parametric analyses; due to the lack of analytic tools, parametric studies tend to consider simplified versions of the algorithm (Clerc and Kennedy 2002).

\section{Conclusions}

Bees, ants, birds, bats, and many other animals have inspired several swarm-based algorithms, but the literature still fails to explain their differences and their complex behavior-a situation that potentially prevents us from understanding and improving such algorithms. In the field, we often describe the differences between the techniques or their versions via the performance achieved when solving distinct problems. This black-box approach has enabled the area to grow over the years and to develop excellent general-use tools. This approach, however, lacks interpretability or explainability. How to interpret, for instance, that including a diversity procedure improves the performance of a swarm algorithm? Is this modification the same as using a different algorithm? With this opaque approach, we miss the opportunity to understand swarm intelligence.

The main barrier to understanding the swarm complex behavior is the discontinuity between the micro-level actions of individuals and the macro-level behavior of the swarm. In our work, we argue that the swarm interaction network is at a meso level that can help to explain and understand these systems. With this approach, we can examine a system via an intermediary structure that emerges from the social interaction within the swarm. 
We can now analyze the patterns of these self-organized interactions. The interaction network also grants an agnostic representation of swarm systems in the swarm interaction space, which provides us with a more general perspective of swarm-based algorithms.

To verify the plausibility of this network-based approach, we considered four different swarm-based algorithms with distinct natural inspirations, and then we focused on one of the most popular optimization techniques, namely, the Particle Swarm Optimization. We also discussed the social interaction in other self-organization mechanisms to guide definitions of their interaction network. In the analysis of the four algorithms, we showed that the interaction network provides us the means to study them from a general perspective. In the in-depth analysis of the PSO algorithm, we found that the interaction network helps us to disentangle complex features of swarm systems. We analyzed its interplay with the quality and improvement of the fitness, and we found that some characteristics of the interaction network can be used to explain parametric settings in the algorithm. Specifically, we studied the diversity in the network (i.e., the Interaction Diversity). Our results revealed that different communication topologies lead the swarm to distinct search modes that also depend on the problem landscape.

The network-based perspective of swarms unfolds a pathway to researchers to study these systems comprehensively. This perspective creates opportunities on two fronts. First, it brings the required general viewpoint to build an objective classification of swarm-based algorithms. This classification guides the algorithm selection for problem-solving and the development of novel or hybrid methods. Second, the network empowers scholars to examine swarms from an intermediate level that is important to understand the complex behavior of these systems. At this meso level, we expose the effects of the swarm rules, which are hidden in the swarm behavior.

From a practical angle, the swarm interaction network brings more transparency to how swarm algorithms work via the perspective of the swarm social interaction. The framework can help in the design of novel algorithms, and it can be used to observe the behavior of an algorithm or a set of algorithms in a specific problem by detecting unexpected situations that can lead to premature converge.

In our research, we have developed a general concept for the interaction network. This definition has limitations that might illuminate research directions. For instance, we lack a procedure to identify the most appropriate description for a given algorithm. Also, we use a static network definition (i.e., a constant number of nodes) that might be inadequate to model some swarm algorithms, especially the ones with evolutionary operators such as selection. In this study, we limited our analyses to optimization algorithms and only performed numerical analyses on the PSO algorithm. Further efforts are needed to investigate the application of the framework on different types of swarm-based algorithms and to identify their fundamental structural characteristics (e.g., motifs, graphlets). Nonetheless, here we proposed a general approach that makes it possible to perform parametric analyses, quantify differences between methods, balance techniques with hybrid or adaptive versions, and build meso-level mechanisms. These are also directions for future research. 


\section{Authors' contributions}

$\mathrm{MO}$ and DP conceived and designed the experiments; $\mathrm{MO}$ and MM performed the experiments; MO, DP, and MM analyzed the data; All authors wrote the paper and have read and approved the final manuscript.

\section{Funding}

This study was financed in part by the Coordenação de Aperfeiçoamento de Pessoal de Nível Superior - Brasil (CAPES) Finance Code 001.

\section{Availability of data and materials}

The code for the analyses performed here can be accessed at http://github.com/macoj/swarm_interaction_network/.

\section{Competing interests}

The authors declare that they have no competing financial interests.

\section{Author details}

${ }^{1}$ Computational Social Science, GESIS-Leibniz Institute for the Social Sciences, Cologne, Germany. ${ }^{2}$ Department of Internal Medicine, University of California, Davis, Sacramento, USA. ${ }^{3}$ Department of Computer Science, University of Exeter, Devon, UK. ${ }^{4}$ Polytechnic School of Pernambuco, University of Pernambuco, Recife, Brazil.

\section{Received: 8 November 2019 Accepted: 18 February 2020}

Published online: 24 May 2020

\section{References}

Abbass HA (2001) A monogenous MBO approach to satisfiability. In: Proceeding of the International Conference on Computational Intelligence for Modelling. CIMCA. pp 1-10

Ali ES, Abd-Elazim SM (2012) Coordinated design of PSSs and TCSC via bacterial swarm optimization algorithm in a multimachine power system. Int J Electr Power Energy Syst 36(1):84-92

Barabási A-L (2012) The network takeover. Nat Phys 8(1):14-16

Barabási A-L, Albert R (1999) Emergence of scaling in random networks. Science 286(5439):509-512

Bastos-Filho CJA, de Lima Neto FB, Lins AJCC, Nascimento AIS, Lima MP (2008) A novel search algorithm based on fish school behavior. In: 2008 IEEE International Conference on Systems, Man and Cybernetics. IEEE, Singapore. pp 2646-2651. https://doi.org/10.1109/icsmc.2008.4811695

Bonabeau E, Dorigo M, Theraulaz G (1999) Swarm intelligence: from natural to artificial systems. Oxford university press

Bourjot C, Chevrier V, Thomas V (2003) A new swarm mechanism based on social spiders colonies: From web weaving to region detection. Web Intell Agent Syst Int J 1(1):47-64

Bratton D, Kennedy J (2007) Defining a standard for particle swarm optimization. In: 2007 IEEE Swarm Intelligence Symposium. IEEE, Honolulu. pp 120-127. https://doi.org/10.1109/sis.2007.368035

Chaté H, Muñoz MA (2014) Viewpoint: insect swarms go critical. Physics 7:120

Chu X, Teresa W, Weir JD, Shi Y, Niu B, Li L (2018) Learning-interaction-diversification. framework for swarm intelligence optimizers: a unified perspective. Neural Comput Appl:1-21. https://doi.org/10.1007/s00521-018-3657-0

Chu S-C, Tsai P-w, Pan J-S (2006) Cat swarm optimization. In: Yang Q, Webb G (eds). PRICAI 2006: Trends in Artificial Intelligence. Springer Berlin Heidelberg, Berlin. pp 854-858

Clerc M (2010) Particle swarm optimization, vol. 93. Wiley

Clerc M, Kennedy J (2002) The particle swarm - explosion, stability, and convergence in a multidimensional complex space. IEEE Trans Evol Comput 6(1):58-73

Dong W, Zhou M (2017) A supervised learning and control method to improve particle swarm optimization algorithms. IEEE Trans Syst Man Cybern Syst 47(7):1135-1148

Dorigo M, Di Caro G (1999) Ant colony optimization: a new meta-heuristic. In: Proceedings of the 1999 Congress on Evolutionary Computation (CEC). IEEE, Washington, DC. pp 1470-1477. https://doi.org/10.1109/cec.1999.782657

Dorigo M, Stützle T (2004) Ant Colony Optimization. The MIT Press

Dorronsoro B, Bouvry P (2012) Study of different small-world topology generation mechanisms for genetic algorithms. In: Proceedings of the 2012 Congress on Evolutionary Computation (CEC). IEEE, Brisbane. pp 10-15. https://doi.org/10. $1109 /$ cec.2012.6256543

Duan H, Luo Q (2015) New progresses in swarm intelligence-based computation. Int J Bio-Inspired Comput 7(1):26

Dynamic Concepts (2014) Cooperative multi-robot control architecture. http://www.dynamic-concepts.com/

Engelbrecht AP (2006) Fundamentals of Computational Swarm Intelligence. Wiley

Engelbrecht AP (2007) Computational Intelligence: An Introduction. 2edition. Wiley Publishing

Engelbrecht AP (2013) Particle swarm optimization: Global best or local best? In: 2013 BRICS Congress on Computational Intelligence and 11 th Brazilian Congress on Computational Intelligence. IEEE, Ipojuca. pp 124-135. https://doi.org/10. 1109/brics-cci-cbic.2013.31

Fehr E, Gächter S (2000) Cooperation and Punishment in Public Goods Experiments. Am Econ Rev 90(4):980-994

Fernandez-Marquez JL, Serugendo GDM, Montagna S, Viroli M, Arcos JL (2012) Description and composition of bio-inspired design patterns: a complete overview. Nat Comput 12(1):43-67

Fewell JH (2003) Social insect networks. Science 301(5641):1867-1870

Fong S, Wang X, Xu Q, Wong R, Fiaidhi J, Mohammed S (2015) Recent advances in metaheuristic algorithms: Does the makara dragon exist? J Supercomput 72(10):3764-3786

Gendreau M, Potvin J-Y (2005) Metaheuristics in combinatorial optimization. Ann Oper Res 140(1):189-213

Giacobini M, Preuss M, Tomassini M (2006) Effects of scale-free and small-world topologies on binary coded self-adaptive cea. In: Lecture Notes in Computer Science (including subseries Lecture Notes in Artificial Intelligence and Lecture Notes in Bioinformatics), vol. 3906 LNCS. Springer, Berlin. pp 86-98 
Godoy A, von Zuben FJ (2009) A complex neighborhood based particle swarm optimization. In: Proceedings of the 2009 Congress on Evolutionary Computation (CEC). IEEE, Trondheim. pp 720-727. https://doi.org/10.1109/cec.2009. 4983016

Havens TC, Spain CJ, Salmon NG, Keller JM (2008) Roach infestation optimization. In: 2008 IEEE Swarm Intelligence Symposium. IEEE, St. Louis. pp 1-7. https://doi.org/10.1109/sis.2008.4668317

Holme P, Saramäki J (2012) Temporal networks. Phys Rep 519(3):97-125

Huepe C, Zschaler G, Do A-L Gross T (2011) Adaptive-network models of swarm dynamics. New J Phys 13(7):073022

Janostik J, Pluhacek M, Senkerik R, Zelinka I (2016) Particle swarm optimizer with diversity measure based on swarm representation in complex network. In: Proceedings of the Second International Afro-European Conference for Industrial Advancement AECIA 2015. Springer, Cham. pp 561-569

Karaboga D (2005) An idea based on honey bee swarm for numerical optimization. Technical report. Computer Engineering Department, Engineering Faculty, Erciyes University, Turkey

Kaufmann S (1993) The origins of order, vol. 209. Oxford University Press

Kennedy J, Eberhart R (1995) Particle swarm optimization. In: Proceedings of ICNN 95 - International Conference on Neural Networks. IEEE, Perth. pp 1942-1948. https://doi.org/10.1109/icnn.1995.488968

Kennedy J, Eberhart RC (2001) Swarm Intelligence. Morgan Kaufmann Publishers Inc.

Krink T, Vesterstrom JS, Riget J (2002) Particle swarm optimisation with spatial particle extension. In: Proceedings of the 2002 Congress on Evolutionary Computation (CEC). IEEE, Honolulu. pp 1474-1479. https://doi.org/10.1109/cec.2002. 1004460

Krishnanand KN, Ghose D (2005) Detection of multiple source locations using a glowworm metaphor with applications to collective robotics. In: Proceedings 2005 IEEE Swarm Intelligence Symposium, 2005. SIS. IEEE, Pasadena. pp 84-91. https://doi.org/10.1109/SIS.2005.1501606

Kromer P, Gajdo P, Zelinka I (2015) Towards a network interpretation of agent interaction in ant colony optimization. In: 2015 IEEE Symposium Series on Computational Intelligence. IEEE, Cape Town. pp 1126-1132. https://doi.org/10. $1109 /$ ssci.2015.161

Lawrence P (1992) The Making of a Fly: the Genetics of Animal Design. Wiley

Liu C, Wen-Bo D, Wang W-X (2014) Particle swarm optimization with scale-free interactions. PLoS ONE 9(5):e97822

Lusseau D (2003) The emergent properties of a dolphin social network. Proc R Soc B: Biol Sci 270:S186—S188

Mamei M, Menezes R, Tolksdorf R, Zambonelli F (2006) Case studies for self-organization in computer science. J Syst Archit 52(8-9):443-460

Meloni S, Xia C-Y, Moreno Y (2017) Heterogeneous resource allocation can change social hierarchy in public goods games. R Soc Open Sci 4(3):170092

Mendes R (2004) Population Topologies and Their Influence in Particle Swarm Performance, PhD thesis. University of Minho

Mendes R, Kennedy J, Neves J (2004) The fully informed particle swarm: Simpler, maybe better. IEEE Trans Evol Comput 8(3):204-210

Metlicka M, Davendra D (2015) Ensemble centralities based adaptive artificial bee algorithm. In: Proceedings of the 2015 Congress on Evolutionary Computation (CEC). IEEE, Sendai. pp 3370-3376. https://doi.org/10.1109/cec.2015.7257312

Monismith DR, Mayfield BE (2008) Slime mold as a model for numerical optimization. In: 2008 IEEE Swarm Intelligence Symposium. SIS. IEEE, St. Louis. pp 1-8. https://doi.org/10.1109/sis.2008.4668295

Moreno JL (1946) Sociogram and sociomatrix. Sociometry 9:348-349

Oliveira M, Bastos-Filho CJA, Menezes R (2013) Assessing particle swarm optimizers using network science metrics. In: Complex Networks IV, volume 476 of Studies in Computational Intelligence. Springer, Berlin. pp 173-184

Oliveira M, Bastos-Filho CJA, Menezes R (2014) Towards a network-based approach to analyze particle swarm optimizers. In: 2014 IEEE Symposium on Swarm Intelligence. IEEE, Orlando. pp 166-173. https://doi.org/10.1109/sis.2014.7011791

Oliveira M, Bastos-Filho CJA, Menezes R (2015) Using network science to assess particle swarm optimizers. Soc Netw Anal Min 5(1):1-13

Oliveira M, Filho CJAB, Menezes R (2013) Using network science to define a dynamic communication topology for particle swarm optimizers. In: Complex Networks. Springer, Berlin. pp 39-47

Oliveira M, Pinheiro D, Andrade B, Bastos-Filho C, Menezes R (2016) Communication Diversity in Particle Swarm Optimizers. Springer International Publishing

Oliveira M, Pinheiro D, Macedo M, Bastos-Filho C, Menezes R (2017) Better exploration-exploitation pace better swarm: Examining the social interactions. In: 2017 IEEE Latin American Conference on Computational Intelligence. LA-CCI. pp $1-6$

Olorunda O, Engelbrecht AP (2008) Measuring exploration/exploitation in particle swarms using swarm diversity. IEEE, Hong Kong. https://doi.org/10.1109/cec.2008.4630938

Parpinelli RS, Lopes HS (2011) New inspirations in swarm intelligence: a survey. Int J Bio-Inspired Comput 3(1):1

Payne JL, Eppstein MJ (2009) Evolutionary dynamics on scale-free interaction networks. IEEE Trans Evol Comput 13(4):895-912

Pluhacek M, Janostik J, Senkerik R, Zelinka I, Davendra D (2016) Pso as complex network—capturing the inner dynamics -initial study. In: Proceedings of the Second International Afro-European Conference for Industrial Advancement. AECIA. Springer, Cham. pp 551-559

Poor RD (2001) Embedded Networks: Pervasive, Low-Power, Wireless Connectivity. PhD thesis, Massachusetts Institute of Technology

Rosenthal SB, Twomey CR, Hartnett AT, Wu HS, Couzin ID (2015) Revealing the hidden networks of interaction in mobile animal groups allows prediction of complex behavioral contagion. Proc Natl Acad Sci 112(15):4690-4695

Schmickl T, Crailsheim K (2007) A navigation algorithm for swarm robotics inspired by slime mold aggregation. In: Swarm Robotics. Springer, Berlin. pp 1-13

Shi Y, Eberhart RC (2008) Population diversity of particle swarms. In: Proceedings of the 2008 Congress on Evolutionary Computation (CEC). IEEE, Hong Kong. pp 1063-1067. https://doi.org/10.1109/cec.2008.4630928

Shi Y, Eberhart R (2009) Monitoring of particle swarm optimization. Front Comput Sci China 3(1):31-37 
Sörensen K (2013) Metaheuristics-the metaphor exposed. Int Trans Oper Res 22(1):3-18

Strandburg-Peshkin A, Twomey CR, Bode NWF, Kao AB, Katz Y, loannou CC, Rosenthal SB, Torney CJ, Wu HS, Levin SA, Couzin ID (2013) Visual sensory networks and effective information transfer in animal groups. Curr Biol 23(17):R709-R711

Strogatz SH (2001) Exploring complex networks. Nature 410(6825):268-276

Sun J, Xiong S-W, Guo F-M (2004) A new pheromone updating strategy in ant colony optimization. In: Proceedings of 2004 International Conference on Machine Learning and Cybernetics. IEEE, Shanghai. pp 620-625. https://doi.org/10. 1109/icmlc.2004.1380766

Tang K, Li X, Suganthan PN, Yang Z, Weise T (2010) Benchmark functions for the CEC'2010 special session and competition on large-scale global optimization. Technical report. Nature Inspired Computation and Applications Laboratory, USTC, China, \& Nanyang Technological University

Vicsek T (2001) A question of scale. Nature 411 (6836):421-421

Watts DJ, Strogatz SH (1998) Collective dynamics of 'small-world' networks. Nature 393(6684):440-442

Wen-Bo D, Ying W, Yan G (2016) The impact of population structure on particle swarm optimization: A network science perspective. In: Lecture Notes in Computer Science. Springer, Cham. pp 341-349

Whitacre JM, Sarker RA, Tuan Pham Q (2008) The self-organization of interaction networks for nature-inspired optimization. IEEE Trans Evol Comput 12(2):220-230

Xia C-Y, Meloni S, Moreno Y (2012) Effects of environment knowledge on agglomeration and cooperation in spatial public goods games. Adv Complex Syst 15(1):1250056

Yang X-S (2009) Firefly algorithm, lévy flights and global optimization. In: Research and Development in Intelligent Systems XXVI. Springer, London. pp 209-218

\section{Publisher's Note}

Springer Nature remains neutral with regard to jurisdictional claims in published maps and institutional affiliations.

Submit your manuscript to a SpringerOpen ${ }^{\circ}$ journal and benefit from:

- Convenient online submission

- Rigorous peer review

Open access: articles freely available online

High visibility within the field

- Retaining the copyright to your article

Submit your next manuscript at $\gg$ springeropen.com 